\title{
SELF-AVOIDING WALKS AND CONNECTIVE CONSTANTS
}

\author{
GEOFFREY R. GRIMMETT AND ZHONGYANG LI
}

\begin{abstract}
The connective constant $\mu(G)$ of a quasi-transitive graph $G$ is the asymptotic growth rate of the number of selfavoiding walks (SAWs) on $G$ from a given starting vertex. We survey several aspects of the relationship between the connective
\end{abstract} constant and the underlying graph $G$.

- We present upper and lower bounds for $\mu$ in terms of the vertex-degree and girth of a transitive graph.

- We discuss the question of whether $\mu \geq \phi$ for transitive cubic graphs (where $\phi$ denotes the golden mean), and we introduce the Fisher transformation for SAWs (that is, the replacement of vertices by triangles).

- We present strict inequalities for the connective constants $\mu(G)$ of transitive graphs $G$, as $G$ varies.

- As a consequence of the last, the connective constant of a Cayley graph of a finitely generated group decreases strictly when a new relator is added, and increases strictly when a non-trivial group element is declared to be a further generator.

- We describe so-called graph height functions within an account of 'bridges' for quasi-transitive graphs, and indicate that the bridge constant equals the connective constant when the graph has a unimodular graph height function.

- A partial answer is given to the question of the locality of connective constants, based around the existence of unimodular graph height functions.

- Examples are presented of Cayley graphs of finitely presented groups that possess graph height functions (that are, in addition, harmonic and unimodular), and that do not.

- The review closes with a brief account of the 'speed' of SAW.

Date: 19 April 2017.

2010 Mathematics Subject Classification. 05C30, 82B20, 60K35.

Key words and phrases. Self-avoiding walk, connective constant, regular graph, transitive graph, quasi-transitive graph, cubic graph, golden mean, Fisher transformation, Cayley graph, bridge constant, locality theorem, graph height function, unimodularity, speed. 


\section{Contents}

1. Introduction

1.1. Self-avoiding walks

1.2. Connective constants, exact values

1.3. Three problems on the square lattice 6

1.4. Critical exponents for SAWs

2. Bounds for connective constants

2.1. Transitivity of graphs

2.2. Bounds for $\mu$ in terms of degree

2.3. Upper bounds for $\mu$ in terms of degree and girth 10

3. Cubic graphs and the golden mean 11

3.1. The Fisher transformation 11

3.2. Bounds for connective constants of cubic graphs 13

4. Strict inequalities for connective constants 14

4.1. Outline of results 14

4.2. Quotient graphs 14

4.3. Quasi-transitive augmentations 17

5. Connective constants of Cayley graphs 18

5.1. Cayley graphs 18

5.2. Strict inequalities for Cayley graphs 18

$\begin{array}{ll}\text { 6. Bridges } & 19\end{array}$

6.1. Bridges and graph height functions 19

6.2. The bridge constant 20

6.3. Weighted Cayley graphs 20

7. Locality of connective constants 21

7.1. Locality of critical values $\quad 21$

7.2. Locality theorem 22

7.3. Application to Cayley graphs 22

8. Existence of graph height functions 23

8.1. Elementary amenable groups 23

8.2. Graphs with no graph height function 23

9. Speed, and the exponent $\nu \quad 24$

$\begin{array}{ll}\text { Acknowledgements } & 27\end{array}$

$\begin{array}{ll}\text { References } & 27\end{array}$ 


\section{INTRODUCTION}

1.1. Self-avoiding walks. A self-avoiding walk (abbreviated to SAW) on a graph $G=(V, E)$ is a path that visits no vertex more than once. An example of a SAW on the square lattice is drawn in Figure 1.1. SAWs were first introduced in the chemical theory of polymerization (see Orr [67] and the book of Flory [22]), and their critical behaviour has attracted the abundant attention since of mathematicians and physicists (see, for example, the book of Madras and Slade [57] and the lecture notes [7]).

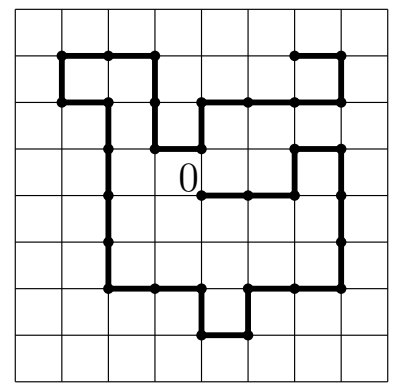

FiguRE 1.1. A 31-step SAW from the origin of the square lattice.

The theory of SAWs impinges on several areas of science including combinatorics, probability, and statistical mechanics. Each of these areas poses its characteristic questions concerning counting and geometry. The most fundamental problem is to count the number of $n$-step SAWs from a given vertex, and this is the starting point of a rich theory of geometry and phase transition.

Let $\sigma_{n}(v)$ be the number of $n$-step SAWs on $G$ starting at the vertex $v$. The following fundamental theorem of Hammersley asserts the existence of an asymptotic growth rate for $\sigma_{n}(v)$ as $n \rightarrow \infty$. (See Section 2.1 for a definition of (quasi-)transitivity.)

Theorem 1.1. [41] Let $G=(V, E)$ be an infinite, connected, quasitransitive graph with finite vertex-degrees. There exists $\mu=\mu(G) \in$ $[1, \infty)$, called the connective constant of $G$, such that

$$
\lim _{n \rightarrow \infty} \sigma_{n}(v)^{1 / n}=\mu, \quad v \in V .
$$

At the heart of the proof is the observation by Hammersley and Morton [42] that (in the case of a transitive graph) $\log \sigma_{n}$ is a subadditive function. That is,

$$
\sigma_{m+n} \leq \sigma_{m} \sigma_{n}, \quad m, n \geq 1
$$


The value $\mu=\mu(G)$ depends evidently on the choice of graph $G$. Indeed, $\mu$ may be viewed as a 'critical point', corresponding, in a sense, to the critical probability of the percolation model, or the critical temperature of the Ising model. Consider the generating function

$$
Z_{v}(x)=\sum_{w \in \Sigma(v)} x^{|w|}, \quad x \in \mathbb{R},
$$

where $\Sigma(v)$ is the set of finite SAWs starting from a given vertex $v$, and $|w|$ is the number of edges of $w$. Viewed as a power series, $Z_{v}(x)$ has radius of convergence $1 / \mu$, and thus a singularity at the point $x=1 / \mu$. Critical exponents may be introduced as in Section 1.4.

In this paper we review certain properties of the connective constant $\mu(G)$, in particular exact values (Section 1.2), upper and lower bounds (Section 2), a sharp lower bound for cubic graphs, and the Fisher transformation (Section 3), strict inequalities (Sections 4-5), and the locality theorem (Section 7). The results summarised here may be found largely in the work of the authors [31]-[36] and [55]. This review is an expanded and updated version of [30].

Previous work on SAWs tends to have been focussed on specific graphs such as the cubic lattices $\mathbb{Z}^{d}$ and certain two-dimensional lattices. In contrast, the results of [31]-[36] are directed at general classes of graphs that are quasi-transitive, and often transitive. The work reviewed here may be the first systematic study of SAWs on general transitive and quasi-transitive graphs. It is useful to have a reservoir of (quasi-)transitive graphs at one's disposal for the construction and analysis of hypotheses, and to this end the Cayley graphs of finitely generated groups play a significant role (see Section 5.1). We note the recent result of Martineau [60] that the set of connective constants of Cayley graphs contains a Cantor space.

Notation for graphs and groups will be introduced when needed. A number of questions are included in this review. The inclusion of a question does not of itself imply either difficulty or importance.

1.2. Connective constants, exact values. For what graphs $G$ is $\mu(G)$ known exactly? There are a number of such graphs, which should be regarded as atypical in this regard. We mention the ladder $\mathbb{L}$, the hexagonal lattice $\mathbb{H}$, and the bridge graph $\mathbb{B}_{\Delta}$ with degree $\Delta \geq 2$ of Figure 1.2, for which

$$
\mu(\mathbb{L})=\frac{1}{2}(1+\sqrt{5}), \quad \mu(\mathbb{H})=\sqrt{2+\sqrt{2}}, \quad \mu\left(\mathbb{B}_{\Delta}\right)=\sqrt{\Delta-1} .
$$

See [2, p. 184] and [19] for the first two calculations. The third is elementary. 

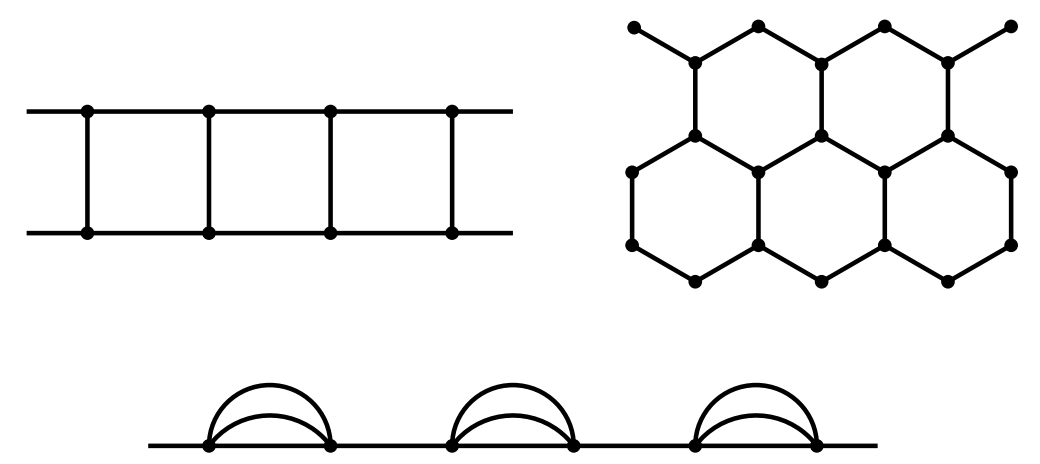

FiguRE 1.2. Three regular graphs: the ladder graph $\mathbb{L}$, the hexagonal tiling $\mathbb{H}$ of the plane, and the bridge graph $\mathbb{B}_{\Delta}$ (with $\Delta=4$ ) obtained from $\mathbb{Z}$ by joining every alternate pair of consecutive vertices by $\Delta-1$ parallel edges.

In contrast, the value of the connective constant of the square grid $\mathbb{Z}^{2}$ is unknown, and a substantial amount of work has been devoted to obtaining good bounds. The best rigorous bounds known currently to the authors are those of $[45,69]$, namely (to 5 significant figures)

$$
2.6256 \leq \mu\left(\mathbb{Z}^{2}\right) \leq 2.6792
$$

and more precise numerical estimates are available, including the estimate $\mu \approx 2.63815 \ldots$ of [44].

We make some remarks about the three graphs of Figure 1.2. There is a correspondence between the Fibonacci sequence and counts of SAWs on the ladder graph $\mathbb{L}$ (see, for example [74]), whereby one obtains that $\mu(\mathbb{L})$ equals the golden ratio $\phi:=\frac{1}{2}(1+\sqrt{5})$. We ask in Question 3.4 whether $\mu(G) \geq \phi$ for all infinite, simple, cubic, transitive graphs, and we discuss evidence for a positive answer to this question.

Amongst a certain class of $\Delta$-regular graphs permitted to possess multiple edges, the bridge graph $\mathbb{B}_{\Delta}=\sqrt{\Delta-1}$ is extremal in the sense that $\mu\left(\mathbb{B}_{\Delta}\right)$ is least. See the discussion of Section 2 .

The proof that $\mu(\mathbb{H})=\sqrt{2+\sqrt{2}}$ by Duminil-Copin and Smirnov [19] is a very significant recent result. The value $\sqrt{2+\sqrt{2}}$ emerged in the physics literature through work of Nienhuis [66] motivated originally by renormalization group theory. Its proof in [19] is based on the construction of an observable with certain properties of discrete holomorphicity, complemented by a neat use of the bridge decomposition 
introduced by Hammersley and Welsh [43]. The bridge decomposition has been used since to prove the locality theorem for connective constants (see Section 6 and Theorem 7.2).

1.3. Three problems on the square lattice. There are a number of beautiful open problems associated with SAWs and connective constants, of which we select three. Our first problem is to prove that a random $n$-step SAW from the origin of $\mathbb{Z}^{2}$ converges, when suitably rescaled, to the Schramm-Loewner curve $\mathrm{SLE}_{8 / 3}$. This important conjecture has been discussed and formalized by Lawler, Schramm, and Werner [52].

Question 1.2. Does a uniformly distributed n-step $S A W$ from the origin of $\mathbb{Z}^{2}$ converge, when suitably rescaled, to the random curve $\mathrm{SLE}_{8 / 3}$ ?

Recent progress in this direction was made by Gwynne and Miller [40], who proved that a SAW on a random quadrangulation converges to $\mathrm{SLE}_{8 / 3}$ on a certain Liouville-gravity surface.

There is an important class of results usually referred to as the "pattern theorem'. In Kesten's original paper [49] devoted to $\mathbb{Z}^{2}$, a proper internal pattern $\mathcal{P}$ is defined as a finite SAW with the property that, for any $k \geq 1$, there exists a SAW containing at least $k$ translates of $\mathcal{P}$. The pattern theorem states that: for a given proper internal pattern $\mathcal{P}$, there exists $a>0$ such that the number of $n$-step SAWs from the origin 0 , containing fewer than an translates of $\mathcal{P}$, is exponentially smaller than the total $\sigma_{n}:=\sigma_{n}(0)$.

The lattice $\mathbb{Z}^{2}$ is bipartite, in that its vertices can be coloured black or white in such a way that every edge links a black vertex and a white vertex. The pattern theorem may be used to prove for this bipartite graph that

$$
\lim _{n \rightarrow \infty} \frac{\sigma_{n+2}}{\sigma_{n}}=\mu^{2}
$$

The proof is based on a surgery of SAWs that preserves the parity of their lengths. The following stronger statement has been open since Kesten's paper [49], see the discussion at [57, p. 244].

Question 1.3. Is it the case for $S A W$ s on $\mathbb{Z}^{2}$ that $\sigma_{n+1} / \sigma_{n} \rightarrow \mu$ ?

Hammersley's Theorem 1.1 establishes the existence of the connective constant for any infinite quasi-transitive graph. It is easy to construct examples of (non-quasi-transitive) graphs for which the limit defining $\mu$ does not exist, and it is natural to enquire of the situation for a random graph. For concreteness, we consider here the infinite cluster $I$ of bond percolation on $\mathbb{Z}^{2}$ with edge-density $p>\frac{1}{2}$ (see [26]). 
Question 1.4. Let $\sigma_{n}(v)$ be the number of n-step SAWs on I starting at the vertex $v$. Does the limit $\mu(v):=\lim _{n \rightarrow \infty} \sigma_{n}(v)^{1 / n}$ exist a.s., and satisfy $\mu(v)=\mu(w)$ a.s. on the event $\{v, w \in I\}$ ?

Discussions of issues around this question, including of when $\mu(v)=$ $p \mu\left(\mathbb{Z}^{2}\right)$ a.s. on the event $\{v \in I\}$, may be found in papers of Lacoin $[50,51]$. The SAW problem on (deterministic) weighted graphs is considered in [38] (see Section 6.3).

1.4. Critical exponents for SAWs. 'Critical exponents' play a significant role in the theory of phase transitions. Such exponents have natural definitions for SAWs on a given graph, as summarised next. The reader is referred to $[7,57]$ and the references therein for general accounts of critical exponents for SAWs. The three exponents that have received most attention in the study of SAWs are as follows.

We consider only the case of SAWs in Euclidean spaces, thus excluding, for example, the hyperbolic space of [59]. Suppose for concreteness that there exists a periodic, locally finite embedding of $G$ into $\mathbb{R}^{d}$ with $d \geq 2$, and no such embedding into $\mathbb{R}^{d-1}$. The case of general $G$ has not been studied extensively, and most attention has been paid to the hypercubic lattice $\mathbb{Z}^{d}$.

The critical exponent $\gamma$. It is believed (when $d \neq 4$ ) that the generic behaviour of $\sigma_{n}(v)$ is given by:

$$
\sigma_{n}(v) \sim A_{v} n^{\gamma-1} \mu^{n}, \quad \text { as } n \rightarrow \infty, \text { for } v \in V,
$$

for constants $A_{v}>0$ and $\gamma \in \mathbb{R}$. The value of the 'critical exponent' $\gamma$ is believed to depend on $d$ only, and not further on the choice of graph $G$. Furthermore, it is believed (and largely proved, see the account in [57]) that $\gamma=1$ when $d \geq 4$. In the borderline case $d=4,(1.5)$ should hold with $\gamma=1$ and subject to the correction factor $(\log n)^{1 / 4}$. (See the related work [6] on weakly self-avoiding walk.)

The critical exponent $\eta$. Let $v, w \in V$, and

$$
Z_{v, w}(x)=\sum_{n=0}^{\infty} \sigma_{n}(v, w) x^{n}, \quad x>0,
$$

where $\sigma_{n}(v, w)$ is the number of $n$-step SAWs with endpoints $v, w$. It is known under certain circumstances that the generating functions $Z_{v, w}$ have radius of convergence $\mu^{-1}$ (see [57, Cor. 3.2.6]), and it is believed that there exists an exponent $\eta$ and constants $A_{v}^{\prime}>0$ such that

$$
Z_{v, w}\left(\mu^{-1}\right) \sim A_{v}^{\prime} d_{G}(v, w)^{-(d-2+\eta)}, \quad \text { as } d_{G}(v, w) \rightarrow \infty,
$$


where $d_{G}(v, w)$ is the graph-distance between $v$ and $w$. Furthermore, $\eta$ satisfies $\eta=0$ when $d \geq 4$.

The critical exponent $\nu$. Let $\Sigma_{n}(v)$ be the set of $n$-step SAWs from $v$, and let $\pi_{n}$ be chosen at random from $\Sigma_{n}(v)$ according to the uniform probability measure. Let $\|\pi\|$ be the graph-distance between the endpoints of a SAW $\pi$. It is believed (when $d \neq 4$ ) that there exists an exponent $\nu$ (the so-called Flory exponent) and constants $A_{v}^{\prime \prime}>0$, such that

$$
\mathbb{E}\left(\left\|\pi_{n}\right\|^{2}\right) \sim A_{v}^{\prime \prime} n^{2 \nu}, \quad v \in V .
$$

As above, this should hold for $d=4$ subject to the inclusion of the correction factor $(\log n)^{1 / 4}$. It is believed that $\nu=\frac{1}{2}$ when $d \geq 4$.

The exponent $\nu$ is an indicator of the geometry of an $n$-step SAW $\pi$ chosen with the uniform measure. In the diffusive case, we have $\nu=\frac{1}{2}$, whereas in the ballistic case (with $\left\|\pi_{n}\right\|$ typically of order $n$ ), we have $\nu=1$. We return to this exponent in Section 9 .

The three exponents $\gamma, \eta, \nu$ are believed to be related through the so-called Fisher relation $\gamma=\nu(2-\eta)$. The definitions (1.5), (1.6), (1.7) may be weakened to logarithmic asymptotics, in which case we say they hold logarithmically.

\section{BOUNDS FOR CONNECTIVE CONSTANTS}

We discuss upper and lower bounds for connective constants in this section, beginning with some algebraic background.

2.1. Transitivity of graphs. The automorphism group of the graph $G=(V, E)$ is denoted $\operatorname{Aut}(G)$, and the identity automorphism is written 1 . The expression $\mathcal{A} \leq \mathcal{B}$ means that $\mathcal{A}$ is a subgroup of $\mathcal{B}$, and $\mathcal{A} \unlhd \mathcal{B}$ means that $\mathcal{A}$ is a normal subgroup.

A subgroup $\Gamma \leq \operatorname{Aut}(G)$ is said to act transitively on $G$ if, for $v, w \in$ $V$, there exists $\gamma \in \Gamma$ with $\gamma v=w$. It is said to act quasi-transitively if there exists a finite set $W$ of vertices such that, for $v \in V$, there exist $w \in W$ and $\gamma \in \Gamma$ with $\gamma v=w$. The graph is called transitive (respectively, quasi-transitive) if $\operatorname{Aut}(G)$ acts transitively (respectively, quasi-transitively) on $G$.

An automorphism $\gamma$ is said to fix a vertex $v$ if $\gamma v=v$. The stabilizer of $v \in V$ is the subgroup

$$
\operatorname{Stab}_{v}:=\{\gamma \in \operatorname{Aut}(G): \gamma v=v\} .
$$

The subgroup $\Gamma$ is said to act freely on $G$ (or on the vertex-set $V$ ) if $\Gamma \cap \operatorname{Stab}_{v}=\{\mathbf{1}\}$ for $v \in V$. 
Let $\mathcal{G}$ (respectively, $\mathcal{Q}$ ) be the set of infinite, simple, locally finite, transitive (respectively, quasi-transitive), rooted graphs, and let $\mathcal{G}_{\Delta}$ (respectively, $\mathcal{Q}_{\Delta}$ ) be the subset comprising $\Delta$-regular graphs. We write $\mathbf{1}=\mathbf{1}_{G}$ for the root of the graph $G$.

2.2. Bounds for $\mu$ in terms of degree. Let $G$ be an infinite, connected, $\Delta$-regular graph. How large or small can $\mu(G)$ be? It is trivial by counting non-backtracking walks that $\sigma_{n}(v) \leq \Delta(\Delta-1)^{n-1}$, whence $\mu(G) \leq \Delta-1$ with equality if $G$ is the $\Delta$-regular tree. It is not difficult to prove the strict inequality $\mu(G)<\Delta-1$ when $G$ is quasi-transitive and contains a cycle (see [33, Thm 4.2]). Lower bounds are harder to obtain.

A multigraph is called loopless if each edge has distinct endvertices.

Theorem 2.1. [33, Thm 4.1] Let $G$ be an infinite, connected, $\Delta$ regular, transitive, loopless multigraph with $\Delta \geq 2$. Then $\mu(G) \geq$ $\sqrt{\Delta-1}$ if either

(a) $G$ is simple, or

(b) $G$ is non-simple and $\Delta \leq 4$.

Note that, for the (non-simple) bridge graph $\mathbb{B}_{\Delta}$ with $\Delta \geq 2$, we have the equality $\mu\left(\mathbb{B}_{\Delta}\right)=\sqrt{\Delta-1}$.

Here is an outline of the proof of Theorem 2.1. A SAW is called forward-extendable if it is the initial segment of some infinite SAW. Let $\sigma_{n}^{\mathrm{F}}(v)$ be the number of forward-extendable $n$-step SAWs starting at $v$. Theorem 2.1 is proved by showing as follows that

$$
\sigma_{2 n}^{\mathrm{F}}(v) \geq(\Delta-1)^{n} .
$$

Let $\pi$ be a (finite) SAW from $v$, with final endpoint $w$. For a vertex $x \in \pi$ satisfying $x \neq w$, and an edge $e \notin \pi$ incident to $x$, the pair $(x, e)$ is called $\pi$-extendable if there exists an infinite SAW starting at $v$ whose initial segment traverses $\pi$ until $x$, and then traverses $e$.

First, it is proved subject to a certain condition $\Pi$ that, for any $2 n$-step forward-extendable SAW $\pi$, there are at least $n(\Delta-2) \pi$ extendable pairs. Inequality (2.1) may be deduced from this statement.

The second part of the proof is to show that graphs satisfying either (a) or (b) of the theorem satisfy condition $\Pi$. It is fairly simple to show that (b) suffices, and it may well be reasonable to extend the conclusion to include values of $\Delta \geq 5$.

Question 2.2. Is it the case that $\mu(G) \geq \sqrt{\Delta-1}$ in the non-simple case of Theorem 2.1(b) with $\Delta \geq 5$ ?

The growth rate $\mu^{\mathrm{F}}$ of the number of forward-extendable SAWs has been studied further by Grimmett, Holroyd, and Peres [29]. They 
show that $\mu^{\mathrm{F}}=\mu$ for any infinite, connected, quasi-transitive graph, with further results involving the numbers of backward-extendable and doubly-extendable SAWs.

Question 2.3. Let $\Delta \geq 3$. What is the sharp lower bound $\mu_{\min }(\Delta):=$ $\inf \left\{\mu(G): G \in \mathcal{G}_{\Delta}\right\}$ ? How does $\mu_{\min }(\Delta)$ behave as $\Delta \rightarrow \infty$ ?

This question is considered in Section 3.2 when $\Delta=3$, and it is asked in Question 3.4 whether or not $\mu_{\min }(3)=\phi$, the golden mean. The lower bound $\mu \geq \sqrt{\Delta-1}$ of Theorem 2.1(a) may be improved as follows when $G$ is non-amenable.

Let $P$ be the transition matrix of simple random walk on $G=(V, E)$, and let $I$ be the identity matrix. The spectral bottom of $I-P$ is defined to be the largest $\lambda$ with the property that, for all $f \in \ell^{2}(V)$,

$$
\langle f,(I-P) f\rangle \geq \lambda\langle f, f\rangle \text {. }
$$

It may be seen that $\lambda(G)=1-\rho(G)$ where $\rho(G)$ is the spectral radius of $P$ (see [56, Sect. 6], and [73] for an account of the spectral radius). It is known that $G$ is a non-amenable if and only if $\rho(G)<1$, which is equivalent to $\lambda(G)>0$. This was proved by Kesten $[47,48]$ for Cayley graphs of finitely-presented groups, and extended to general transitive graphs by Dodziuk [16] (see also the references in [56, Sect. 6.10]).

Theorem 2.4. [36, Thm 6.2] Let $G \in \mathcal{G}_{\Delta}$ with $\Delta \geq 3$, and let $\lambda=\lambda(G)$ be the above spectral bottom. The connective constant satisfies

$$
\mu(G) \geq(\Delta-1)^{\frac{1}{2}(1+c \lambda)},
$$

where $c=\Delta(\Delta-1) /(\Delta-2)^{2}$.

2.3. Upper bounds for $\mu$ in terms of degree and girth. The girth of a simple graph is the length of its shortest cycle. Let $\mathcal{G}_{\Delta, g}$ be the subset of $\mathcal{G}_{\Delta}$ containing graphs with girth $g$.

Theorem 2.5. [34, Thm 7.4] For $G \in \mathcal{G}_{\Delta, g}$ where $\Delta, g \geq 3$, we have that $\mu(G) \leq y$ where $\zeta:=1 / y$ is the smallest positive real root of the equation

$$
(\Delta-2) \frac{M_{1}(\zeta)}{1+M_{1}(\zeta)}+\frac{M_{2}(\zeta)}{1+M_{2}(\zeta)}=1,
$$

with

$$
M_{1}(\zeta)=\zeta, \quad M_{2}(\zeta)=2\left(\zeta+\zeta^{2}+\cdots+\zeta^{g-1}\right) .
$$

The upper bound $y$ is sharp, and is achieved by the free product graph $F:=K_{2} * K_{2} * \cdots * K_{2} * \mathbb{Z}_{g}$, with $\Delta-2$ copies of the complete graph $K_{2}$ on two vertices and one copy of the cycle $\mathbb{Z}_{g}$ of length $g$. 
The proof follows quickly by earlier results of Woess [73], and Gilch and Müller [24]. By [73, Thm 11.6], every $G \in \mathcal{G}_{\Delta, g}$ is covered by $F$, and by [24, Thm 3.3], $F$ has connective constant $1 / \zeta$.

\section{CUbiC GRAPHS AND THE GOLDEN MEAN}

A graph is called cubic if it is regular with degree $\Delta=3$. Cubic graphs have the property that every edge-self-avoiding cycle is also vertex-self-avoiding. We assume throughout this section that $G=$ $(V, E) \in \mathcal{Q}_{3}$, and we write $\phi:=\frac{1}{2}(1+\sqrt{5})$ for the golden mean.

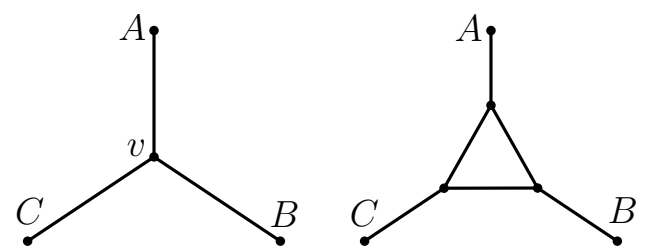

FiguRE 3.1. The Fisher transformation of the star.

3.1. The Fisher transformation. Let $v \in V$, and recall that $v$ has degree 3 by assumption. The so-called Fisher transformation acts at $v$ by replacing it by a triangle, as illustrated in Figure 3.1. The Fisher transformation has been valuable in the study of the relations between Ising, dimer, and general vertex models (see [13, 21, 53, 54]), and also in the calculation of the connective constant of the Archimedean lattice $\left(3,12^{2}\right)$ (see, for example, $\left.[28,39,46]\right)$. The Fisher transformation may be applied at every vertex of a cubic graph, of which the hexagonal and square/octagon lattices are examples.

Let $G$ be, in addition, quasi-transitive. By Theorem 1.1, $G$ has a well-defined connective constant $\mu=\mu(G)$ satisfying (1.1). Write $F(G)$ for the graph obtained by applying the Fisher transformation at every vertex of $G$. The automorphism group of $G$ induces an automorphism subgroup of $F(G)$, so that $F(G)$ is quasi-transitive and has a well-defined connective constant. It is noted in [31], and probably elsewhere also, that the connective constants of $G$ and $F(G)$ have a simple relationship. This conclusion, and its iteration, are given in the next theorem

Theorem 3.1. [31, Thm 3.1] Let $G \in \mathcal{Q}_{3}$, and consider the sequence $\left(G_{k}: k=0,1,2, \ldots\right)$ given by $G_{0}=G$ and $G_{k+1}=F\left(G_{k}\right)$.

(a) The connective constants $\mu_{k}:=\mu\left(G_{k}\right)$ satisfy $\mu_{k}^{-1}=g\left(\mu_{k+1}^{-1}\right)$ where $g(x)=x^{2}+x^{3}$. 


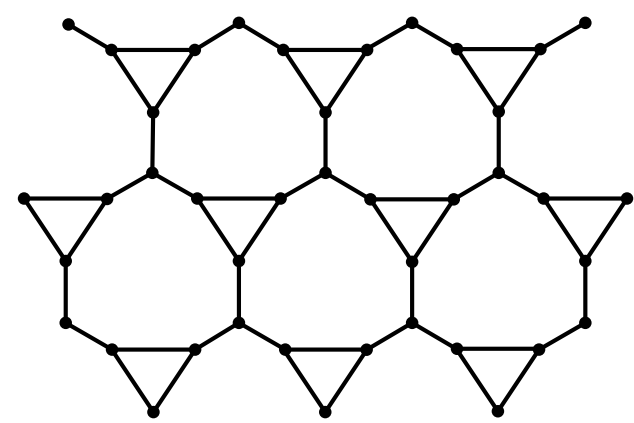

Figure 3.2. The lattice $\widetilde{\mathbb{H}}$ is derived from the hexagonal lattice $\mathbb{H}$ by applying the Fisher transformation at alternate vertices. Its connective constant $\widetilde{\mu}$ is a root of the equation $x^{-3}+x^{-4}=1 /(2+\sqrt{2})$.

(b) The sequence $\mu_{k}$ converges monotonely to the golden mean $\phi$, and

$$
-\left(\frac{4}{7}\right)^{k} \leq \mu_{k}^{-1}-\phi^{-1} \leq\left(\frac{2}{7-\sqrt{5}}\right)^{k}, \quad k \geq 1 .
$$

The idea underlying part (a) is that, at each vertex $v$ visited by a SAW $\pi$ on $G_{k}$, one may replace that vertex by either of the two paths around the 'Fisher triangle' of $G_{k+1}$ at $v$. Some book-keeping is necessary with this argument, and this is best done via the generating functions (1.3).

A similar argument may be applied in the context of a 'semi-cubic' graph.

Theorem 3.2. [31, Thm 3.3] Let $G$ be an infinite, connected, bipartite graph with vertex-sets coloured black and white, and suppose the coloured graph is quasi-transitive, and every black vertex has degree 3. Let $\widetilde{G}$ be the graph obtained by applying the Fisher transformation at each black vertex. The connective constants $\mu$ and $\widetilde{\mu}$ of $G$ and $\widetilde{G}$, respectively, satisfy $\mu^{-2}=h\left(\widetilde{\mu}^{-1}\right)$, where $h(x)=x^{3}+x^{4}$.

Example 3.3. Take $G=\mathbb{H}$, the hexagonal lattice with connective constant $\mu=\sqrt{2+\sqrt{2}} \approx 1.84776$, see [19]. The ensuing lattice $\widetilde{\mathbb{H}}$ is illustrated in Figure 3.2, and its connective constant $\widetilde{\mu}$ satisfies $\mu^{-2}=$ $h\left(\widetilde{\mu}^{-1}\right)$, which may be solved to obtain $\widetilde{\mu} \approx 1.75056$.

We return briefly to the critical exponents of Section 1.4. In [31, Sect. 3], reasonable definitions of the three exponents $\gamma, \eta, \nu$ are presented, 
none of which depend on the existence of embeddings into $\mathbb{R}^{d}$. Furthermore, it is proved that the values of the exponents are unchanged under the Fisher transformation.

3.2. Bounds for connective constants of cubic graphs. Amongst cubic graphs, the 3-regular tree $T_{3}$ has largest connective constant $\mu\left(T_{3}\right)=2$. It is an open problem to determine the sharp lower bound on $\mu(G)$ for $G \in \mathcal{G}_{3}$. Recall the ladder graph $\mathbb{L}$ of Figure 1.2, with $\mu(\mathbb{L})=\phi$.

Question 3.4. [34, Qn 1.1] Is it the case that $\mu(G) \geq \phi$ for $G \in \mathcal{G}_{3}$ ?

Even in the case of graphs with small girth, the best general lower bounds known so far are as follows.

Theorem 3.5. [34, Thms 7.1, 7.2]

(a) For $G \in \mathcal{G}_{3,3}$, we have that

$$
\mu(G) \geq x,
$$

where $x \in(1,2)$ satisfies

$$
\frac{1}{x^{2}}+\frac{1}{x^{3}}=\frac{1}{\sqrt{2}}
$$

(b) For $G \in \mathcal{G}_{3,4}$, we have that

$$
\mu(G) \geq 12^{1 / 6} .
$$

The sharp upper bounds for $\mathcal{G}_{3,3}$ and $\mathcal{G}_{3,4}$ are those of Theorem 2.5, and they are attained respectively by the Fisher graph of the 3-regular tree, and of the degree- 4 tree (in which each vertex is replaced by a 4-cycle).

Question 3.4 is known to have a positive answer for various classes of graph, including so-called TLF-planar graphs (see [34, 70]). The word plane means a simply connected Riemann surface without boundaries. An embedding of a graph $G=(V, E)$ in a plane $\mathcal{P}$ is a function $\eta: V \cup$ $E \rightarrow \mathcal{P}$ such that $\eta$ restricted to $V$ is an injection and, for $e=\langle u, v\rangle \in$ $E, \eta(e)$ is a $C^{1}$ image of $[0,1]$. An embedding is $\left(\mathcal{P}_{-}\right)$planar if the images of distinct edges are disjoint except possibly at their endpoints, and a graph is $\left(\mathcal{P}_{-}\right)$planar if it possesses a $\left(\mathcal{P}_{-}\right)$planar embedding. An embedding is topologically locally finite $(T L F)$ if the images of the vertices have no accumulation point, and a connected graph is called $T L F$-planar if it possesses a planar TLF embedding. Let $\mathcal{T}_{\Delta}$ denote the class of transitive, TLF-planar graphs with vertex-degree $\Delta$.

Theorem 3.6. [34] Let $G \in \mathcal{T}_{3}$ be infinite. Then $\mu(G) \geq \phi$. 
Two techniques are used repeatedly in the proof. The first is to construct an injection from the set of SAWs on the ladder graph $\mathbb{L}$ that move either rightwards or vertically, into the set of SAWs on a graph $G^{\prime}$ derived from $G$. A large subclass of $\mathcal{T}_{3}$ may be treated using such a construction. The remaining graphs in $\mathcal{T}_{3}$ require detailed analyses using a variety of transformations of graphs including the Fisher transformation of Section 3.1.

A second class of graphs for which $\mu \geq \phi$ is given as follows. The definition of a transitive graph height function is deferred to Definition 6.1 .

Theorem 3.7. [34, Thm 3.1] We have that $\mu(G) \geq \phi$ for any cubic graph $G \in \mathcal{G}_{3}$ that possesses a transitive graph height function.

This theorem covers all Cayley graphs of finitely presented groups with strictly positive first Betti numbers (see Section 5.1 and [34, Example 3]). Cayley graphs are introduced in Section 5.1.

\section{Strict inequalities For COnNective COnstants}

4.1. Outline of results. Consider a probabilistic model on a graph $G$, such as the percolation or random-cluster model (see [27]). There is a parameter (perhaps 'density' $p$ or 'temperature' $T$ ) and a 'critical point' (usually written $p_{\mathrm{c}}$ or $T_{\mathrm{c}}$ ). The numerical value of the critical point depends on the choice of graph $G$. It is often important to understand whether a systematic change in the graph causes a strict change in the value of the critical point. A general approach to this issue was presented by Aizenman and Grimmett [1] and developed further in $[5,12,25]$ and [26, Chap. 3]. The purpose of this section is to review work of [32] directed at the corresponding question for self-avoiding walks.

Let $G$ be a subgraph of $G^{\prime}$, and suppose each graph is quasi-transitive. It is trivial that $\mu(G) \leq \mu\left(G^{\prime}\right)$. Under what conditions does the strict inequality $\mu(G)<\mu\left(G^{\prime}\right)$ hold? Two sufficient conditions for the strict inequality are reviewed here. This is followed in Section 5 with a summary of consequences for Cayley graphs.

The results of this section apply to transitive graphs. Difficulties arise under the weaker assumption of quasi-transitivity.

4.2. Quotient graphs. Let $G=(V, E) \in \mathcal{G}$. Let $\Gamma \leq \operatorname{Aut}(G)$ act transitively, and let $\mathcal{A} \unlhd \Gamma$ (we shall discuss the non-normal case later). There are several ways of constructing a quotient graph $G / \mathcal{A}$, the strongest of which (for our purposes) is given next. The set of neighbours of a vertex $v \in V$ is denoted by $\partial v$. 
We denote by $\vec{G}=(\bar{V}, \vec{E})$ the directed quotient graph $G / \mathcal{A}$ constructed as follows. Let $\approx$ be the equivalence relation on $V$ given by $v_{1} \approx v_{2}$ if and only if there exists $\alpha \in \mathcal{A}$ with $\alpha v_{1}=v_{2}$. The vertexset $\bar{V}$ comprises the equivalence classes of $(V, \approx)$, that is, the orbits $\bar{v}:=\mathcal{A} v$ as $v$ ranges over $V$. For $v, w \in V$, we place $|\partial v \cap \bar{w}|$ directed edges from $\bar{v}$ to $\bar{w}$ (if $\bar{v}=\bar{w}$, these edges are directed loops).

Example 4.1. Let $G$ be the square lattice $\mathbb{Z}^{2}$ and let $m \geq 1$. Let $\Gamma$ be the set of translations of $\mathbb{Z}^{2}$, and let $\mathcal{A}$ be the normal subgroup of $\Gamma$ generated by the map that sends $(i, j)$ to $(i+m, j)$. The quotient graph $G / \mathcal{A}$ is the square lattice 'wrapped around a cylinder', with each edge replaced by two oppositely directed edges.

Since $\vec{G}$ is obtained from $G$ by a process of identification of vertices and edges, it is natural to ask whether the strict inequality $\mu(\vec{G})<$ $\mu(G)$ is valid. Sufficient conditions for this strict inequality are presented next.

Let $L=L(G, \mathcal{A})$ be the length of the shortest SAW of $G$ with (distinct) endpoints in the same orbit. Thus, for example, $L=1$ if $\vec{G}$ possesses a directed loop. A group is called trivial if it comprises the identity only.

Theorem 4.2. [32, Thm 3.8] Let $\Gamma$ act transitively on $G$, and let $\mathcal{A}$ be a non-trivial, normal subgroup of $\Gamma$. The connective constant $\vec{\mu}=\mu(\vec{G})$ satisfies $\vec{\mu}<\mu(G)$ if: either

(a) $L \neq 2$, or

(b) $L=2$ and either of the following holds:

(i) $G$ contains some 2 -step $S A W v\left(=w_{0}\right), w_{1}, w_{2}\left(=v^{\prime}\right)$ satisfying $\bar{v}=\bar{v}^{\prime}$ and $\left|\partial v \cap \bar{w}_{1}\right| \geq 2$,

(ii) $G$ contains some $S A W v\left(=w_{0}\right), w_{1}, w_{2}, \ldots, w_{l}\left(=v^{\prime}\right)$ satisfying $\bar{v}=\bar{v}^{\prime}, \bar{w}_{i} \neq \bar{w}_{j}$ for $0 \leq i<j<l$, and furthermore $v^{\prime}=\alpha v$ for some $\alpha \in \mathcal{A}$ which fixes no $w_{i}$.

Remark 4.3. In the situation of Theorem 4.2, can one calculate an explicit $R=R(G, \mathcal{A})<1$ such that $\mu(\vec{G}) / \mu(G)<R$ ? The answer is (in principle) positive under a certain condition, namely that the socalled 'bridge constant' of $G$ equals its connective constant. Bridges are discussed in Section 6, and it is shown in Theorem 6.2 that the above holds when $G$ possesses a so-called 'unimodular graph height function' (see Definition 6.1). See also [32, Thm 3.11] and [37, Remark 4.5].

We call $\mathcal{A}$ symmetric if

$$
|\partial v \cap \bar{w}|=|\partial w \cap \bar{v}|, \quad v, w \in V .
$$


Consider the special case $L=2$ of Theorem 4.2. Condition (i) of Theorem 4.2(b) holds if $\mathcal{A}$ is symmetric, since $|\partial w \cap \bar{v}| \geq 2$. Symmetry of $\mathcal{A}$ is implied by unimodularity, for a definition of which we refer the reader to [32, Sect. 3.5] or [56, Sect. 8.2].

Example 4.4. Conditions (i)-(ii) of Theorem 4.2(b) are necessary in the case $L=2$, in the sense illustrated by the following example. Let $G$ be the infinite 3-regular tree with a distinguished end $\omega$. Let $\Gamma$ be the set of automorphisms that preserve $\omega$, and let $\mathcal{A}$ be the normal subgroup generated by the interchanges of the two children of any given vertex $v$ (and the associated relabelling of their descendants). The graph $\vec{G}$ is isomorphic to that obtained from $\mathbb{Z}$ by replacing each edge by two directed edges in one direction and one in the reverse direction. It is easily seen that $L=2$, but that neither (i) nor (ii) holds. Indeed, $\mu(\vec{G})=\mu(G)=2$.

The proof of Theorem 4.2 follows partly the general approach of Kesten in his pattern theorem, see [49] and [57, Sect. 7.2]. Any $n$-step SAW $\vec{\pi}$ in the directed graph $\vec{G}$ lifts to a SAW $\pi$ in the larger graph $G$. The idea is to show there exists $a>0$ such that 'most' such $\vec{\pi}$ contain at least an sub-SAWs for which the corresponding sub-walks of $\pi$ may be replaced by SAWs on $G$. Different subsets of these sub-SAWs of $\vec{G}$ give rise to different SAWs on $G$. The number of such subsets grows exponentially in $n$, and this introduces an exponential 'entropic' factor in the counts of SAWs.

Unlike Kesten's proof and its later elaborations, these results apply in the general setting of transitive graphs, and they utilize algebraic and combinatorial techniques.

We discuss next the assumption of normality of $\mathcal{A}$ in Theorem 4.2. The (undirected) simple quotient graph $\bar{G}=(\bar{V}, \bar{E})$ may be defined as follows even if $\mathcal{A}$ is not a normal subgroup of $\Gamma$. As before, the vertex-set $\bar{V}$ is the set of orbits of $V$ under $\mathcal{A}$. Two distinct orbits $\mathcal{A} v$, $\mathcal{A} w$ are declared adjacent in $\bar{G}$ if there exist $v^{\prime} \in \mathcal{A} v$ and $w^{\prime} \in \mathcal{A} w$ with $\left\langle v^{\prime}, w^{\prime}\right\rangle \in E$. We write $\bar{G}=G_{\mathcal{A}}$ to emphasize the role of $\mathcal{A}$.

The relationship between the site percolation critical points of $G$ and $G_{\mathcal{A}}$ is the topic of a conjecture of Benjamini and Schramm [10], which appears to make the additional assumption that $\mathcal{A}$ acts freely on $V$. The last assumption is stronger than the assumption of unimodularity.

We ask for an example in which the non-normal case is essentially different from the normal case.

Question 4.5. Let $\Gamma$ be a subgroup of $\operatorname{Aut}(G)$ acting transitively on $G$. Can there exist a non-normal subgroup $\mathcal{A}$ of $\Gamma$ such that: (i) the 
quotient graph $G_{\mathcal{A}}$ is transitive, and (ii) there exists no normal subgroup $\mathcal{N}$ of some transitively acting $\Gamma^{\prime}$ such that $G_{\mathcal{A}}$ is isomorphic to $G_{\mathcal{N}}$ ? Might it be relevant to assume that $\mathcal{A}$ acts freely on $V$ ?

We return to connective constants with the following question, inspired in part by [10].

Question 4.6. Is it the case that $\mu\left(G_{\mathcal{A}}\right)<\mu(G)$ under the assumption that $\mathcal{A}$ is a non-trivial (not necessarily normal) subgroup of $\Gamma$ acting freely on $V$, such that $G_{\mathcal{A}}$ is transitive?

The proof of Theorem 4.2 may be adapted to give an affirmative answer to Question 4.6 subject to a certain extra condition on $\mathcal{A}$, see [32, Thm 3.12]. Namely, it suffices that there exists $l \in \mathbb{N}$ such that $G_{\mathcal{A}}$ possesses a cycle of length $l$ but $G$ has no cycle of this length.

4.3. Quasi-transitive augmentations. We consider next the systematic addition of new edges, and the effect thereof on the connective constant. Let $G=(V, E) \in \mathcal{G}$. From $G$, we derive a second graph $G^{\prime}=\left(V, E^{\prime}\right)$ by adding further edges to $E$, possibly in parallel to existing edges. We assume that $E$ is a proper subset of $E^{\prime}$.

Theorem 4.7. [32, Thm 3.2] Let $\Gamma \leq \operatorname{Aut}(G)$ act transitively on $G$, and let $\mathcal{A} \leq \Gamma$ satisfy either or both of the following.

(a) $\mathcal{A}$ is a normal subgroup of $\Gamma$ acting quasi-transitively on $G$.

(b) The index $[\Gamma: A]$ is finite.

If $\mathcal{A} \leq \operatorname{Aut}\left(G^{\prime}\right)$, then $\mu(G)<\mu\left(G^{\prime}\right)$.

Example 4.8. Let $\mathbb{Z}^{2}$ be the square lattice, with $\mathcal{A}$ the group of its translations. The triangular lattice $\mathbb{T}$ is obtained from $\mathbb{Z}^{2}$ by adding the edge $e=\langle 0,(1,1)\rangle$ together with its images under $\mathcal{A}$, where 0 denotes the origin. Since $\mathcal{A}$ is a normal subgroup of itself, it follows that $\mu\left(\mathbb{Z}^{2}\right)<\mu(\mathbb{T})$. This example may be extended to augmentations by other periodic families of new edges, as explained in [32, Example 3.4].

Remark 4.9. In the situation of Theorem 4.7, can one calculate an $R>1$ such that $\mu\left(G^{\prime}\right) / \mu(G)>R$ ? As in Remark 4.3, the answer is positive when $G^{\prime}$ has a unimodular graph height function.

A slightly more general form of Theorem 4.7 is presented in [32]. Can one dispense with the assumption of normality in Theorem 4.7(a)?

Question 4.10. Let $\Gamma$ act transitively on $G$, and let $\mathcal{A}$ be a subgroup of $\Gamma$ that acts quasi-transitively on $G$. If $\mathcal{A} \leq \operatorname{Aut}\left(G^{\prime}\right)$, is it necessarily the case that $\mu(G)<\mu\left(G^{\prime}\right)$ ? 
A positive answer would be implied by an affirmative answer to the following question.

Question 4.11. Let $G \in \mathcal{G}$, and let $\mathcal{A} \leq \operatorname{Aut}(G)$ act quasi-transitively on $G$. When does there exist a subgroup $\Gamma$ of $\operatorname{Aut}(G)$ acting transitively on $G$ such that $\mathcal{A} \leq \Gamma$ and $\Gamma \cap \operatorname{Stab}_{v} \leq \mathcal{A}$ for $v \in V$ ?

See [32, Prop. 3.6] and the further discussion therein.

\section{Connective constants of Cayley graphs}

5.1. Cayley graphs. Let $\Gamma$ be an infinite group with identity element $\mathbf{1}$ and finite generator-set $S$, where $S$ satisfies $S=S^{-1}$ and $\mathbf{1} \notin S$. Thus, $\Gamma$ has a presentation as $\Gamma=\langle S \mid R\rangle$ where $R$ is a set of relators. The group $\Gamma$ is called finitely generated since $|S|<\infty$, and finitely presented if, in addition, $|R|<\infty$

The Cayley graph $G=G(\Gamma, S)$ is defined as follows. The vertexset $V$ of $G$ is the set of elements of $\Gamma$. Distinct elements $g, h \in V$ are connected by an edge if and only if there exists $s \in S$ such that $h=g s$. It is easily seen that $G$ is connected, and $\Gamma$ acts transitively by left-multiplication. It is standard that $\Gamma$ acts freely, and hence $G$ is unimodular and therefore symmetric. Accounts of Cayley graphs may be found in [4] and [56, Sect. 3.4].

Reference is occasionally made here to the first Betti number of $\Gamma$. This is the power of $\mathbb{Z}$, denoted $B(\Gamma)$, in the abelianization $\Gamma /[\Gamma, \Gamma]$. (See [35, Remark 4.2].)

5.2. Strict inequalities for Cayley graphs. Theorems 4.2 and 4.7 have the following implications for Cayley graphs. Let $s_{1} s_{2} \cdots s_{l}=\mathbf{1}$ be a relation. This relation corresponds to the closed walk $\left(\mathbf{1}, s_{1}, s_{1} s_{2}, \ldots\right.$, $s_{1} s_{2} \cdots s_{l}=\mathbf{1}$ ) of $G$ passing through the identity $\mathbf{1}$. Consider now the effect of adding a further relator. Let $t_{1}, t_{2}, \ldots, t_{l} \in S$ be such that $\rho:=t_{1} t_{2} \cdots t_{l}$ satisfies $\rho \neq \mathbf{1}$, and write $\Gamma_{\rho}=\langle S \mid R \cup\{\rho\}\rangle$. Then $\Gamma_{\rho}$ is isomorphic to the quotient group $\Gamma / \mathcal{N}$ where $\mathcal{N}$ is the normal subgroup of $\Gamma$ generated by $\rho$.

Theorem 5.1. [32, Corollaries 4.1, 4.3] Let $G=G(\Gamma, S)$ be the Cayley graph of the infinite, finitely presented group $\Gamma=\langle S \mid R\rangle$.

(a) Let $G_{\rho}=G\left(\Gamma_{\rho}, S\right)$ be the Cayley graph obtained by adding to $R$ a further non-trivial relator $\rho$. Then $\mu\left(G_{\rho}\right)<\mu(G)$.

(b) Let $w \in \Gamma$ satisfy $w \neq 1, w \notin S$, and let $\bar{G}_{w}$ be the Cayley graph of the group obtained by adding $w$ (and $w^{-1}$ ) to $S$. Then $\mu(G)<\mu\left(\bar{G}_{w}\right)$. 
As noted in Remarks 4.3 and 4.9, non-trivial bounds may in principle be calculated for the ratios of the two connective constants in case (a) (respectively, case (b)) whenever $G$ (respectively, $\bar{G}_{w}$ ) has a unimodular graph height function.

Example 5.2. The square/octagon lattice, otherwise known as the Archimedean lattice $\left(4,8^{2}\right)$, is the Cayley graph of the group with generator set $S=\left\{s_{1}, s_{2}, s_{3}\right\}$ and relator set

$$
R=\left\{s_{1}^{2}, s_{2}^{2}, s_{3}^{2}, s_{1} s_{2} s_{1} s_{2}, s_{1} s_{3} s_{2} s_{3} s_{1} s_{3} s_{2} s_{3}\right\} .
$$

(See [32, Fig. 3].) By adding the further relator $s_{2} s_{3} s_{2} s_{3}$, we obtain a graph isomorphic to the ladder graph of Figure 1.2, whose connective constant is the golden mean $\phi$.

By Theorem 5.1(a), the connective constant $\mu$ of the square/octagon lattice is strictly greater than $\phi=1.618 \ldots$ The best lower bound currently known appears to be $\mu>1.804 \ldots$, see [45].

Example 5.3. The square lattice $\mathbb{Z}^{2}$ is the Cayley graph of the abelian group with $S=\{a, b\}$ and $R=\left\{a b a^{-1} b^{-1}\right\}$. We add a generator $a b$ (and its inverse), thus adding a diagonal to each square of $\mathbb{Z}^{2}$. Theorem 5.1(b) implies the standard inequality $\mu\left(\mathbb{Z}^{2}\right)<\mu(\mathbb{T})$ of Example 4.8.

\section{BRIDGES}

6.1. Bridges and graph height functions. Various surgical constructions are useful in the study of self-avoiding walks, of which the most elementary involves concatenations of so-called 'bridges'. Bridges were introduced by Hammersley and Welsh [43] in the context of the hypercubic lattice $\mathbb{Z}^{d}$. An $n$-step bridge on $\mathbb{Z}^{d}$ is a self-avoiding walk $\pi=\left(\pi_{0}, \pi_{1}, \ldots, \pi_{n}\right)$ such that

$$
\pi_{0}(1)<\pi_{m}(1) \leq \pi_{n}(1), \quad 0<m \leq n,
$$

where $x(1)$ denotes the first coordinate of a vertex $x \in \mathbb{Z}^{d}$.

The significant property of bridges is as follows: given two bridges $\pi=\left(0, x_{1}, \ldots, x_{m}\right), \pi^{\prime}=\left(0, y_{1}, \ldots, y_{n}\right)$ starting at 0 , the concatenation $\pi \cup\left[x_{m}+\pi^{\prime}\right]$ is an $(m+n)$-step bridge from 0 . It follows that the number $b_{n}$ of $n$-step bridges from 0 satisfies

$$
b_{m+n} \geq b_{m} b_{n}
$$

whence the bridge constant $\beta\left(\mathbb{Z}^{d}\right):=\lim _{n \rightarrow \infty} b_{n}^{1 / n}$ exists. Since $b_{n} \leq \sigma_{n}$, it is trivial that $\beta\left(\mathbb{Z}^{d}\right) \leq \mu\left(\mathbb{Z}^{d}\right)$. Using a surgery argument, Hammersley and Welsh proved amongst other things that $\beta=\mu$ for $\mathbb{Z}^{d}$. 
In this section, we discuss the bridge constant for transitive graphs, therein introducing the graph height functions that will be useful in the discussion of locality in Section 7.

First we define a graph height function, and then we use such a function to define a bridge.

Definition 6.1. [37, Defn 3.1] Let $G=(V, E) \in \mathcal{Q}$ with root labelled 1 .

(i) A graph height function on $G$ is a pair $(h, \mathcal{H})$ such that:

(a) $h: V \rightarrow \mathbb{Z}$, and $h(\mathbf{1})=0$,

(b) $\mathcal{H} \leq \operatorname{Aut}(G)$ acts quasi-transitively on $G$ such that $h$ is $\mathcal{H}$-difference-invariant, in the sense that

$$
h(\alpha v)-h(\alpha u)=h(v)-h(u), \quad \alpha \in \mathcal{H}, u, v \in V,
$$

(c) for $v \in V$, there exist $u, w \in \partial v$ such that $h(u)<h(v)<$ $h(w)$.

(ii) A graph height function $(h, \mathcal{H})$ is called transitive (respectively, unimodular) if the action of $\mathcal{H}$ is transitive (respectively, unimodular).

The reader is referred to [56, Chap. 8] and [37, eqn (3.1)] for discussions of unimodularity.

6.2. The bridge constant. Let $(h, \mathcal{H})$ be a graph height function of the graph $G \in \mathcal{Q}$. A bridge $\pi=\left(\pi_{0}, \pi_{1}, \ldots, \pi_{n}\right)$ is a SAW on $G$ satisfying

$$
h\left(\pi_{0}\right)<h\left(\pi_{m}\right) \leq h\left(\pi_{n}\right), \quad 0<m \leq n .
$$

Let $b_{n}$ be the number of $n$-step bridges $\pi$ from $\pi_{0}=1$. Using quasitransitivity, it may be shown (similarly to (6.1)) that the limit $\beta=$ $\lim _{n \rightarrow \infty} b_{n}^{1 / n}$ exists, and $\beta$ is called the bridge constant. Note that $\beta$ depends on the choice of graph height function.

The following is proved by an extension of the methods of [43].

Theorem 6.2. [37, Thm 4.3] Let $G \in \mathcal{Q}$ possess a unimodular graph height function $(h, \mathcal{H})$. The associated bridge constant $\beta=\beta(G, h, \mathcal{H})$ satisfies $\beta=\mu(G)$.

In particular, the value of $\beta$ does not depend on the choice of unimodular graph height function.

6.3. Weighted Cayley graphs. A natural extension of the theory of self-avoiding walks is to edge-weighted graphs. Let $G=(V, E)$ be 
an infinite graph, and let $\phi: E \rightarrow[0, \infty)$. The weight of a SAW $\pi$ traversing the edges $e_{1}, e_{2}, \ldots, e_{n}$ is defined as

$$
w_{\phi}(\pi):=\prod_{i=1}^{n} \phi\left(e_{i}\right) .
$$

One may ask about the asymptotic behaviour of the sum of the $w_{\phi}(\pi)$ over all SAWs $\pi$ with length $n$ starting at a given vertex. The question is more interesting when $G$ is not assumed locally finite, since the number $\sigma_{n}$ of SAWs from a given vertex may then be infinite. Some conditions are needed on the pair $(G, \phi)$, and these are easiest stated when $G$ is a Cayley graph.

Let $\Gamma=\langle S \mid R\rangle$ be an infinite, finitely presented group, with a Cayley graph $G$ (we do not assume that $G$ is locally finite). Let $\phi: \Gamma \rightarrow[0, \infty)$ be such that

(a) $\phi(\mathbf{1})=0$,

(b) $\phi$ is symmetric in that $\phi(\gamma)=\phi\left(\gamma^{-1}\right)$ for $\gamma \in \Gamma$,

(c) $\phi$ is summable in that $\sum_{\gamma \in \Gamma} \phi(\gamma)<\infty$.

The aggregate weights of SAWs on $G$ have been studied in [38]. It turns out to be useful to consider a generalized notion of the length $l(\pi)$ of a SAW $\pi$, and there is an interaction between $l$ and $\phi$. Subject to certain assumptions (in particular, $\Gamma$ is assumed virtually indicable), it is shown that the bridge and connective constants are equal. This yields a continuity theorem for the connective constants of weighted graphs.

\section{LoCAlity of CONNECTIVE CONSTANTS}

7.1. Locality of critical values. The locality question for SAWs may be stated as follows: for which families of rooted graphs is the value of the connective constant $\mu=\mu(G)$ determined by the graph-structure of large bounded neighbourhoods of the root of $G$ ? Similar questions have been asked for other systems including the percolation model, see $[9,61]$.

Let $G \in \mathcal{Q}$. The ball $S_{k}=S_{k}(G)$, with radius $k$, is the rooted subgraph of $G$ induced by the set of its vertices within distance $k$ of the root 1 . For $G, G^{\prime} \in \mathcal{Q}$, we write $S_{k}(G) \simeq S_{k}\left(G^{\prime}\right)$ if there exists a graph-isomorphism from $S_{k}(G)$ to $S_{k}\left(G^{\prime}\right)$ that maps $\mathbf{1}$ to $\mathbf{1}^{\prime}$. Let

$$
K\left(G, G^{\prime}\right)=\max \left\{k: S_{k}(G) \simeq S_{k}\left(G^{\prime}\right)\right\}, \quad G, G^{\prime} \in \mathcal{G},
$$

and $d\left(G, G^{\prime}\right)=2^{-K\left(G, G^{\prime}\right)}$. The corresponding metric space was introduced by Babai [3]; see also [11, 15]. 
Question 7.1. Under what conditions on $G \in \mathcal{Q}$ and $\left\{G_{n}\right\} \subseteq \mathcal{Q}$ is it the case that

$$
\mu\left(G_{n}\right) \rightarrow \mu(G) \text { if } K\left(G, G_{n}\right) \rightarrow \infty ?
$$

The locality property of connective constants turns out to be related in a surprising way to the existence of harmonic graph height functions (see Theorem 8.1).

7.2. Locality theorem. Let $G \in \mathcal{Q}$ support a graph height function $(h, \mathcal{H})$. There are two associated integers $d, r$ defined as follows. Let

$$
d=d(h)=\max \{|h(u)-h(v)|: u, v \in V, u \sim v\} .
$$

If $\mathcal{H}$ acts transitively, we set $r=0$. Assume $\mathcal{H}$ does not act transitively, and let $r=r(h, \mathcal{H})$ be the infimum of all $r$ such that the following holds. Let $o_{1}, o_{2}, \ldots, o_{M}$ be representatives of the orbits of $\mathcal{H}$. For $i \neq j$, there exists $v_{j} \in \mathcal{H} o_{j}$ such that $h\left(o_{i}\right)<h\left(v_{j}\right)$, and a SAW from $o_{i}$ to $v_{j}$, with length $r$ or less, all of whose vertices $x$, other than its endvertices, satisfy $h\left(o_{i}\right)<h(x)<h\left(v_{j}\right)$.

For $D \geq 1$ and $R \geq 0$, let $\mathcal{Q}_{D, R}$ be the subset of $\mathcal{Q}$ containing graphs which possess a unimodular graph height function $(h, \mathcal{H})$ with $d(h) \leq D$ and $r(h, \mathcal{H}) \leq R$.

Theorem 7.2 (Locality theorem). [37, Thm 5.1] Let $G \in \mathcal{Q}$. Let $D \geq 1$ and $R \geq 0$, and let $G_{n} \in \mathcal{Q}_{D, R}$ for $n \geq 1$. If $K\left(G, G_{n}\right) \rightarrow \infty$ as $n \rightarrow \infty$, then $\mu\left(G_{n}\right) \rightarrow \mu(G)$.

The rationale of the proof is as follows. Consider for simplicity the case of transitive graphs. Since $\log \sigma_{n}$ is a subadditive sequence (see (1.2)), we have that $\mu \leq \sigma_{n}^{1 / n}$ for $n \geq 1$. Similarly, by (6.1), $\log \beta_{n}$ is superadditive, so that $\beta \geq b_{n}^{1 / n}$ for $n \geq 1$. Therefore,

$$
b_{n}^{1 / n} \leq \beta \leq \mu \leq \sigma_{n}^{1 / n}, \quad n \geq 1 .
$$

Now, $b_{n}$ and $\sigma_{n}$ depend only on the ball $S_{n}(G)$. If $G$ is such that $\beta=\mu$, then their shared value can be approximated, within any prescribed accuracy, by counts of bridges and SAWs on bounded balls. By Theorem 6.2 , this holds if $G$ supports a unimodular graph height function.

7.3. Application to Cayley graphs. Let $\Gamma=\langle S \mid R\rangle$ be finitely presented with Cayley graph $G=G(\Gamma, S)$. Let $t \in \Gamma$ have infinite order. We present an application of the Locality Theorem 7.2 to the situation in which a new relator $t^{n}$ is added. Let $G_{n}$ be the Cayley graph of the group $\Gamma_{n}=\left\langle S \mid R \cup\left\{t^{n}\right\}\right\rangle$.

Theorem 7.3. [35, Thm 6.1] If the first Betti number of $\Gamma$ satisfies $B(\Gamma) \geq 2$, then $\mu\left(G_{n}\right) \rightarrow \mu(G)$ as $n \rightarrow \infty$. 


\section{Existence OF GRAPH HEIGHT FUnCTIONS}

We saw in Sections 6 and 7 that, subject to the existence of certain unimodular graph height functions, the equality $\beta=\mu$ holds, and a locality result follows. In addition, there exists a terminating algorithm for calculating $\mu$ to any degree of precision (see [37]). In this section, we identify certain classes of graphs that possess unimodular graph height functions.

For simplicity in the following, we restrict ourselves to Cayley graphs of finitely generated groups.

8.1. Elementary amenable groups. The class EG of elementary amenable groups was introduced by Day in 1957, [14], as the smallest class of groups that contains the set $\mathrm{EG}_{0}$ of all finite and abelian groups, and is closed under the operations of taking subgroups, and of forming quotients, extensions, and directed unions. Day noted that every group in EG is amenable (see also von Neumann [65]). Let EFG be the set of infinite, finitely generated members of EG.

Theorem 8.1. [36, Thm 4.1] Let $\Gamma \in$ EFG. There exists a normal subgroup $\mathcal{H} \unlhd \Gamma$ with $[\Gamma: \mathcal{H}]<\infty$ such that any locally finite Cayley graph $G$ of $\Gamma$ possesses a graph height function of the form $(h, \mathcal{H})$ which is both unimodular and harmonic.

Note that the graph height function $(h, \mathcal{H})$ of the theorem is harmonic. It has a further property, namely that $\mathcal{H} \unlhd \Gamma$ has finite index, and $\mathcal{H}$ acts on $\Gamma$ by left-multiplication. Such a graph height function is called strong.

The proof of Theorem 8.1 has two stages. Firstly, by a standard algebraic result, there exist $\mathcal{H} \unlhd \Gamma$ such that: $|\Gamma / \mathcal{H}|<\infty$, and $\mathcal{H}$ is indicable in that there exists a surjective homomorphism $F: \mathcal{H} \rightarrow \mathbb{Z}$. At the second stage, we consider a random walk on the Cayley graph $G$, and set $h(\gamma)=\mathbb{E}_{\gamma}(F(H))$, where $H$ is the first hitting point of $\mathcal{H}$ viewed as a subset of vertices. That $h$ is harmonic off $\mathcal{H}$ is automatic, and on $\mathcal{H}$ because the action of $\mathcal{H}$ is unimodular.

The conclusion of Theorem 8.1 is in fact valid for the larger class of infinite, finitely generated, virtually indicable groups (see [38, Thm $3.2])$.

8.2. Graphs with no graph height function. There exist transitive graphs possessing no graph height function, and examples include the (amenable) Cayley graph of the Grigorchuk group, and the (nonamenable) Cayley graph of the Higman group (see [36, Thms 5.1, 8.1]). This may be deduced from the next theorem. 
Theorem 8.2. [36, Cor. 9.2] Let $\Gamma=\langle S \mid R\rangle$ where $|S|<\infty$, and let $\Pi$ be the subgroup of permutations of $S$ that preserve $\Gamma$ up to isomorphism. Let $G$ be a Cayley graph of $\Gamma$ satisfying Stab $_{1}=\Pi$, where $\Pi$ is viewed as a subgroup of $\operatorname{Aut}(G)$.

(a) If $\Gamma$ is a torsion group, then $G$ has no graph height function.

(b) Suppose $\Gamma$ has no proper, normal subgroup with finite index. If $G$ has graph height function $(h, \mathcal{H})$, then $(h, \Gamma)$ is also a graph height function.

The point is that, when $\operatorname{Stab}_{1}=\Pi$, every automorphism of $G$ is obtained by a certain composition of an element of $\Gamma$ and an element of $\Pi$. The Grigorchuk group is a torsion group, and part (a) applies. The Higman group $\Gamma$ satisfies part (b), and is quickly seen to have no graph height function of the form $(h, \Gamma)$. (A graph height function of the form $(h, \Gamma)$ is called a group height function in [35, Sect. 4].)

\section{Speed, And the exponent $\nu$}

For simplicity in this section, we consider only transitive rooted graphs $G$. Let $\pi_{n}$ be a random $n$-step SAW from the root of $G$, chosen according to the uniform measure on the set $\Sigma_{n}$ of such walks. What can be said about the graph-distance $\left\|\pi_{n}\right\|$ between the endpoints of $\pi_{n}$ ?

We say that SAW on $G$ has positive speed if there exist $c, \alpha>0$ such that

$$
\mathbb{P}\left(\left\|\pi_{n}\right\| \leq c n\right) \leq e^{-\alpha n}, \quad n \geq 0 .
$$

While stronger than the natural definition through the requirement of exponential decay to 0 , this is a useful definition for the results of this section. When $G$ is infinite, connected, and quasi-transitive, and SAW on $G$ has positive speed, it is immediate that

$$
C n^{2} \leq \mathbb{E}\left(\left\|\pi_{n}\right\|^{2}\right) \leq n^{2},
$$

for some $C>0$; thus (1.7) holds (in a slightly weaker form) with $\nu=1$.

For SAW on $\mathbb{Z}^{d}$ it is known, [18], that SAW does not have positive speed, and that that $\mathbb{E}\left(\left\|\pi_{n}\right\|^{2}\right) / n^{2} \rightarrow 0$ as $n \rightarrow \infty$ (cf. (1.7)). Complementary 'delocalization' results have been proved in [17], for example that, for $\epsilon>0$ and large $n$,

$$
\mathbb{P}\left(\left\|\pi_{n}\right\|=1\right) \leq n^{-\frac{1}{4}+\epsilon},
$$

and it is asked there whether

$$
\mathbb{P}\left(\left\|\pi_{n}\right\|=x\right) \leq n^{-\frac{1}{4}+\epsilon}, \quad x \in \mathbb{Z}^{d} .
$$

We pose the following question for non-amenable graphs. 
Question 9.1. [18] Is it the case that, for any non-amenable Cayley graph $G$ of an infinite, finitely generated group, $S A W$ on $G$ has positive speed?

Progress towards this question may be summarised as follows. By bounding the number of SAWs by the number of non-backtracking paths, Nachmias and Peres [64, eqn (2.3)] have proved that that, for a non-amenable, transitive graph $G$ satisfying

$$
(\Delta-1) \rho<\mu
$$

SAW on $G$ has positive speed. Here, $\Delta$ is the vertex degree, $\rho$ is the spectral radius of simple random walk on $G$ (see the discussion around (2.2)), and $\mu$ is the connective constant.

It is classical (see, for example, $\left[26\right.$, eqn (1.13)]) that $\mu p_{\mathrm{c}} \geq 1$, where $p_{\mathrm{c}}$ is the critical probability of bond percolation on $G$. Inequality (9.1) is therefore implied by the stronger inequality

$$
(\Delta-1) \rho p_{\mathrm{c}}<1
$$

These inequalities (9.1)-(9.2) are useful in several settings.

A. [64] There exist $\rho_{0}<1$ and $g_{0}<\infty$ such that, if $G$ is an non-amenable, transitive graph with spectral radius less than $\rho_{0}$ and girth at least $g_{0}$, then (9.2) holds, and hence SAW on $G$ has positive speed.

B. [68] Let $S \subset \Gamma$ be a finite symmetric generating set of an infinite group $\Gamma$, and $\Delta=|S|$. Let $S^{(k)}$ be the multiset of cardinality $\Delta^{k}$ comprising all elements $g \in \Gamma$ with length not exceeding $k$ in the word metric given by $S$, each such element included with multiplicity equal to the number of such ways of expressing $g$. The set $S^{(k)}$ generates $\Gamma$. By [68, Proof of Thm 2],

$$
\rho\left(G, S^{(k)}\right) p_{\mathrm{c}}\left(G, S^{(k)}\right) \Delta^{k} \rightarrow 0 \quad \text { as } k \rightarrow \infty,
$$

where $\left(G, S^{(k)}\right)$ denotes the (possibly non-simple) Cayley graph of $\Gamma$ with generator-set $S^{(k)}$. Inequality (9.2) follows for sufficiently large $k$

C. By the argument of [68, Prop. 1], (9.2) holds if

$$
\rho<\frac{\Delta^{2}+\Delta-1}{\Delta^{2}+(\Delta-1)^{2}}
$$

This holds when $\rho<\frac{1}{2}$, irrespective of $\Delta$.

D. Thom [72] has shown that, for any finitely generated, nonamenable group $\Gamma$ and any $\epsilon>0$, there exists a finite symmetric generating set $S$ such that the corresponding Cayley graph 
$G=G(\Gamma, S)$ has $\rho<\epsilon$. By the above, SAW on $G$ has positive speed.

E. [71] Let $i=i(G)$ be the edge-isoperimetric constant of an infinite, transitive graph $G$. Since $p_{\mathrm{c}} \leq(1+i)^{-1}$ and $\rho^{2} \leq 1-(i / \Delta)^{2}$ ([62, Thm 2.1(a)]), inequality (9.2) holds whenever

$$
(\Delta-1) \frac{\sqrt{1-(i / \Delta)^{2}}}{1+i}<1 .
$$

It is sufficient that $i / \Delta>1 / \sqrt{2}$.

F. [20] It is proved that

$$
\rho \leq \frac{\sqrt{8 \Delta-16}+3.47}{\Delta}
$$

when $G$ is planar. When combined with $\mathrm{C}$ above, for example, this implies that SAW has positive speed on any transitive, planar graph with sufficiently large $\Delta$. A related inequality for hyperbolic tesselations is found in [20, Thm 7.4].

We turn next to graphs embedded in the hyperbolic plane. It was proved by Madras and $\mathrm{Wu}$ [58] that SAWs on most regular tilings of the hyperbolic plane have positive speed. Note that the graphs treated in [58] are both (vertex-)transitive and edge-transitive (unlike the graphs in $\mathrm{F}$ above). It was proved by Benjamini [8] that SAWs on the seven regular planar triangulations of the hyperbolic plane have mean displacement bounded beneath by a linear function.

We turn finally to a discussion of the number of ends of a transitive graph. The number of ends of an infinite, connected graph $G=(V, E)$ is the supremum over all finite subsets $W \subset V$ of the number of infinite components that remain after deletion of $W$. An infinite, finitely generated group $\Gamma$ is said to have $k$ ends if some locally finite Cayley graph (and hence all such Cayley graphs) has $k$ ends. Recall from [63, Prop. 6.2] that a transitive graph $G$ has $k \in\{1,2, \infty\}$ and, moreover, if $k=2$ (respectively, $k=\infty$ ) then $G$ is amenable (respectively, non-amenable).

Infinite, connected, (quasi-)transitive graphs with two or more ends have been studied by $\mathrm{Li}$ [55, Thm 1.3], who has proved, subject to two conditions, that SAW has positive speed. The approach of the proof (see [55]) is to consider a finite 'cutset' $W$ with the property that many SAWs cross $S$ to another component of $G \backslash W$ and never cross back. The pattern theorem is a key element in the proof. These results may be applied, for example, to a cylindrical quotient graph of $\mathbb{Z}^{d}$ (see $[2,23])$, and the infinite free product of two finite, transitive, connected graphs. Here are two corollaries for Cayley graphs. 
Theorem 9.2. [55, Thms 1.7, 1.8] Let $\Gamma$ be an infinite, finitely generated group with two or more ends.

(a) If $\Gamma$ has infinitely many ends, and $G$ is a locally finite Cayley graph, there exists $c>0$ such that

$$
\limsup _{n \rightarrow \infty}\left|\left\{\pi \in \Sigma_{n}(\mathbf{1}):\|\pi\| \geq c n\right\}\right|^{1 / n}=\mu .
$$

(b) There exists some locally finite, Cayley graph $G$ such that $S A W$ on $G$ has positive speed.

\section{ACKNowledgements}

This work was supported in part by the Engineering and Physical Sciences Research Council under grant EP/I03372X/1. GRG acknowledges valuable conversations with Alexander Holroyd concerning Questions 4.5 and 4.11, and the hospitality of UC Berkeley during the completion of the work. ZL acknowledges support from the Simons Foundation under grant \#351813, and the National Science Foundation under grant DMS-1608896.

\section{REFERENCES}

[1] M. Aizenman and G. R. Grimmett, Strict monotonicity of critical points in percolation and ferromagnetic models, J. Statist. Phys. 63 (1991), 817-835.

[2] S. E. Alm and S. Janson, Random self-avoiding walks on one-dimensional lattices, Commun. Statist. Stoch. Mod. 6 (1990), 169-212.

[3] L. Babai, Vertex-transitive graphs and vertex-transitive maps, J. Graph Th. 15 (1991), 587-627.

[4] _ Automorphism groups, isomorphism, reconstruction, Handbook of Combinatorics, vol. II, Elsevier, Amsterdam, 1995, pp. 1447-1540.

[5] P. Balister, B. Bollobás, and O. Riordan, Essential enhancements revisited, (2014), https://arxiv.org/abs/1402.0834.

[6] R. Bauerschmidt, D. C. Brydges, and G. Slade, Renormalisation group analysis of $4 D$ spin models and self-avoiding walk, Proceedings ICMP, Santiago de Chile, 2015, https://arxiv.org/abs/1602.04048.

[7] R. Bauerschmidt, H. Duminil-Copin, J. Goodman, and G. Slade, Lectures on self-avoiding walks, Probability and Statistical Physics in Two and More Dimensions (D. Ellwood, C. M. Newman, V. Sidoravicius, and W. Werner, eds.), Clay Mathematics Institute Proceedings, vol. 15, CMI/AMS publication, 2012, pp. 395-476.

[8] I. Benjamini, Self avoiding walk on the seven regular triangulations, (2016), https://arxiv.org/abs/1612.04169.

[9] I. Benjamini, A. Nachmias, and Y. Peres, Is the critical percolation probability local?, Probab. Th. Rel. Fields 149 (2011), 261-269.

[10] I. Benjamini and O. Schramm, Percolation beyond $\mathbb{Z}^{d}$, many questions and a few answers, Electron. Commun. Probab. 1 (1996), 71-82. 
[11] _ Recurrence of distributional limits of finite planar graphs, Electron. J. Probab. 6 (2001), Article 23.

[12] C. Bezuidenhout, G. R. Grimmett, and H. Kesten, Strict inequality for critical values of Potts models and random-cluster processes, Commun. Math. Phys. 158 (1993), 1-16.

[13] C. Boutillier and B. de Tilière, The critical Z-invariant Ising model via dimers: locality property, Commun. Math. Phys. 301 (2011), 473-516.

[14] M. M. Day, Amenable semigroups, Illinois J. Math. 1 (1957), 509-544.

[15] R. Diestel and I. Leader, A conjecture concerning a limit of non-Cayley graphs, J. Alg. Combin. 14 (2001), 17-25.

[16] J. Dodziuk, Difference equations, isoperimetric inequality and transience of certain random walks, Trans. Amer. Math. Soc. 284 (1984), 787-794.

[17] H. Duminil-Copin, A. Glazman, A. Hammond, and Manolescu I., On the probabiity that self-avoiding walk ends at a given point, Ann. Probab. 44 (2016), 955-983.

[18] H. Duminil-Copin and A. Hammond, Self-avoiding walk is sub-ballistic, Commun. Math. Phys. 324 (2013), 401-423.

[19] H. Duminil-Copin and S. Smirnov, The connective constant of the honeycomb lattice equals $\sqrt{2+\sqrt{2}}$, Ann. Math. 175 (2012), 1653-1665.

[20] Z. Dvorak and B. Mohar, Spectral radius of finite and infinite planar graphs and of graphs of bounded genus, J. Combin. Theory Ser. B 100 (2010), 729-739.

[21] M. E. Fisher, On the dimer solution of planar Ising models, J. Math. Phys. 7 (1966), 1776-1781.

[22] P. Flory, Principles of Polymer Chemistry, Cornell University Press, 1953.

[23] H. Frauenkron, M. S. Causo, and P. Grassberger, Two-dimensional selfavoiding walks on a cylinder, Phys. Rev. E 59 (1999), R16-R19.

[24] L. A. Gilch and S. Müller, Counting self-avoiding walks on free products of graphs, Discrete Math. 340 (2017), 325-332.

[25] G. R. Grimmett, Potts models and random-cluster processes with many-body interactions, J. Statist. Phys. 75 (1994), 67-121.

[26] _ Percolation, 2nd ed., Springer, Berlin, 1999.

[27] _ The Random-Cluster Model, Springer, Berlin, 2006, http://www. statslab.cam.ac.uk/ grg/books/rcm.html.

[28] - Three theorems in discrete random geometry, Probab. Surv. 8 (2011), 403-441.

[29] G. R. Grimmett, A. E. Holroyd, and Y. Peres, Extendable self-avoiding walks, Ann. Inst. Henri Poincaré D 1 (2014), 61-75.

[30] G. R. Grimmett and Z. Li, Counting self-avoiding walks, (2013), https:// arxiv.org/abs/1304.7216.

[31] _ Self-avoiding walks and the Fisher transformation, Electron. J. Combin. 20 (2013), Paper 47, 14 pp.

[32] __ Strict inequalities for connective constants of transitive graphs, SIAM J. Discrete Math. 28 (2014), 1306-1333.

[33] Bounds on the connective constants of regular graphs, Combinatorica 35 (2015), 279-294.

[34] , Cubic graphs and the golden mean, (2016), https://arxiv.org/abs/ 1610.00107. 
[35] _ Connective constants and height functions for Cayley graphs, Trans. Amer. Math. Soc. 369 (2017), 5961-5980.

[36] _ Self-avoiding walks and amenability, Electron. J. Combin. 24 (2017), Paper P4.38, 24 pp.

[37] _ Locality of connective constants, Discrete Math. 341 (2018), 34833497.

[38] _ Weighted self-avoiding walks, J. Alg. Combin. (2019), https:// arxiv.org/abs/1804.05380.

[39] A. J. Guttmann, R. Parviainen, and A. Rechnitzer, Self-avoiding walks and trails on the $3.12^{2}$ lattice, J. Phys. A: Math. Gen. 38 (2005), 543-554.

[40] E. Gwynne and J. Miller, Convergence of the self-avoiding walk on random quadrangulations to $S L E_{8 / 3}$ on $\sqrt{8 / 3}$-Liouville quantum gravity, (2016), https://arxiv.org/abs/1608.00956.

[41] J. M. Hammersley, Percolation processes II. The connective constant, Proc. Camb. Phil. Soc. 53 (1957), 642-645.

[42] J. M. Hammersley and W. Morton, Poor man's Monte Carlo, J. Roy. Statist. Soc. B 16 (1954), 23-38.

[43] J. M. Hammersley and D. J. A. Welsh, Further results on the rate of convergence to the connective constant of the hypercubical lattice, Quart. J. Math. Oxford 13 (1962), 108-110.

[44] I. Jensen, A parallel algorithm for the enumeration of self-avoiding polygons on the square lattice, J. Phys. A: Math. Gen. 36 (2003), 5731-5745.

[45] _ Improved lower bounds on the connective constants for twodimensional self-avoiding walks, J. Phys. A: Math. Gen. 37 (2004), 1152111529 .

[46] I. Jensen and A. J. Guttman, Self-avoiding walks, neighbour-avoiding walks and trails on semiregular lattices, J. Phys. A: Math. Gen. 31 (1998), 8137-8145.

[47] H. Kesten, Full Banach mean values on countable groups, Math. Scand. 7 (1959), 146-156.

[48] , Symmetric random walks on groups, Trans. Amer. Math. Soc. 92 (1959), 336-354.

[49] _ On the number of self-avoiding walks, J. Math. Phys. 4 (1963), 960969.

[50] H. Lacoin, Existence of a non-averaging regime for the self-avoiding walk on a high-dimensional infinite percolation cluster, J. Statist. Phys. 154 (2014), $1461-1482$.

[51] _ Non-coincidence of quenched and annealed connective constants on the supercritical planar percolation cluster, Probab. Th. Rel. Fields 159 (2014), $777-808$.

[52] G. Lawler, O. Schramm, and W. Werner, On the scaling limit of planar selfavoiding walk, Proc. Sympos. Pure Math. 72 (2004), 339-364.

[53] Z. Li, Local statistics of realizable vertex models, Commun. Math. Phys. 304 (2011), 723-763.

[54] Critical temperature of periodic Ising models, Commun. Math. Phys. 315 (2012), 337-381.

[55] _ Positive speed self-avoiding walks on graphs with more than one end, (2016), https://arxiv.org/abs/1612.02464. 
[56] R. Lyons and Y. Peres, Probability on Trees and Networks, Cambridge University Press, New York, 2016, http://mypage.iu.edu/ rdlyons/.

[57] N. Madras and G. Slade, Self-Avoiding Walks, Birkhäuser, Boston, 1993.

[58] N. Madras and C. Wu, Self-avoiding walks on hyperbolic graphs, Combin. Probab. Comput. 14 (2005), 523-548.

[59] N. Madras and C. C. Wu, Self-avoiding walks on hyperbolic graphs, Combin. Probab. Comput. 14 (2005), 523-548.

[60] S. Martineau, The set of connective constants of Cayley graphs contains a Cantor space, Electron. Commun. Probab. 22 (2017), Paper No. 12.

[61] S. Martineau and V. Tassion, Locality of percolation for Abelian Cayley graphs, Ann. Probab. 45 (2017), 1247-1277.

[62] B. Mohar, Isoperimetric inequalities, growth, and spectrum of graphs, Lin. Alg. Appl. 103 (1988), 119-131.

[63] _ Some relations between analytic and geometric properties of infinite graphs, Discrete Math. 95 (1991), 193-219, Directions in infinite graph theory and combinatorics (Cambridge, 1989).

[64] A. Nachmias and Y. Peres, Non-amenable Cayley graphs of high girth have $p_{c}<p_{u}$ and mean-field exponents, Electron. Commun. Probab. 17 (2012), $1-8$.

[65] J. von Neumann, Zur allgemeinen Theorie des Masses, Fund. Math. 13 (1929), $73-116$.

[66] B. Nienhuis, Exact critical points and critical exponents of $\mathrm{O}(n)$ models in two dimensions, Phys. Rev. Lett. 49 (1982), 1062-1065.

[67] W. J. C. Orr, Statistical treatment of polymer solutions at infinite dilution, Trans. Faraday Soc. 43 (1947), 12-27.

[68] I. Pak and T. Smirnova-Nagnibeda, On non-uniqueness of percolation on nonamenable Cayley graphs, C. R. Acad. Sci. Paris 33 (2000), 495-500.

[69] A. Pönitz and P. Tittmann, Improved upper bounds for self-avoiding walks on $\mathbb{Z}^{d}$, Electron. J. Combin. 7 (2000), paper R21.

[70] D. Renault, The vertex-transitive TLF-planar graphs, Discrete Math. 309 (2009), 2815-2833.

[71] R. H. Schonmann, Multiplicity of phase transitions and mean-field criticality on highly non-amenable graphs, Commun. Math. Phys. 219 (2001), 271-322.

[72] A. Thom, A remark about the spectral radius, Int. Math. Res. Notices 10 (2015), 2856-2864.

[73] W. Woess, Random Walks on Infinite Graphs and Groups, Cambridge University Press, Cambridge, 2000.

[74] D. Zeilberger, Self-avoiding walks, the language of science, and Fibonacci numbers, J. Statist. Plann. Inference 54 (1996), 135-138.

Statistical Laboratory, Centre for Mathematical Sciences, CamBridge University, Wilberforce Road, CAmbridge CB3 0WB, UK

E-mail address: g.r.grimmett@statslab.cam.ac.uk

URL: http://www.statslab.cam.ac.uk/ grg/ 
Department of Mathematics, University of Connecticut, 341 MansFIELd RoAd U1009, Storrs, CT 06269-1009, USA

E-mail address: zhongyang.li@uconn.edu

URL: http://www. math. uconn.edu/ zhongyang/ 